\title{
Multi-user Collisions: Applications to Discrete Logarithm, Even-Mansour and PRINCE
}

\author{
Pierre-Alain Fouque ${ }^{1}$, Antoine Joux ${ }^{2}$, and Chrysanthi Mavromati ${ }^{3}$ \\ 1 Université Rennes 1, France and Institut Universitaire de France, France \\ 2 CryptoExperts, France and Chaire de Cryptologie de la Fondation de l'UPMC \\ Laboratoire d'Informatique de Paris 6, UPMC Sorbonne Universités, France \\ 3 Sogeti/ESEC R\&D Lab, France \\ Université de Versailles Saint-Quentin-en-Yvelines, France \\ pierre-alain.fouque@univ-rennes1.fr, antoine.joux@m $4 \mathrm{x}$. org, \\ chrysanthi.mavromati@sogeti.com
}

\begin{abstract}
In this paper, we investigate the multi-user setting both in public and in secret-key cryptanalytic applications. In this setting, the adversary tries to recover keys of many users in parallel more efficiently than with classical attacks, i.e., the number of recovered keys multiplied by the time complexity to find a single key, by amortizing the cost among several users. One possible scenario is to recover a single key in a large set of users more efficiently than to recover a key in the classical model. Another possibility is, after some shared precomputation, to be able to learn individual keys very efficiently. This latter model is close to traditional time/memory tradeoff attacks with precomputation. With these goals in mind, we introduce two new algorithmic ideas to improve collision-based attacks in the multi-user setting. Both ideas are derived from the parallelizable collision search as proposed by van Oorschot and Wiener. This collision search uses precomputed chains obtained by iterating some basic function. In our cryptanalytic application, each pair of merging chains can be used to correlate the key of two distinct users. The first idea is to construct a graph, whose vertices are keys and whose edges are these correlations. When the graph becomes connected, we simultaneously recover all the keys. Thanks to random graph analysis techniques, we can show that the number of edges that are needed to make this event occurs is small enough to obtain some improved attacks. The second idea modifies the basic technique of van Oorschot and Wiener: instead of waiting for two chains to merge, we now require that they become parallel.

We first show that, using the first idea alone, we can recover the discrete logarithms of $L$ users in a group of size $N$ in time $\widetilde{O}(\sqrt{N L})$. We put these two ideas together and we show that in the multi-user Even-Mansour scheme, all the keys of $L=N^{1 / 3}$ users can be found with $N^{1 / 3+\epsilon}$ queries for each user (where $N$ is the domain size). Finally, we consider the PRINCE block cipher (with 128-bit keys and 64-bit blocks) and find the keys of 2 users among a set of $2^{32}$ users in time $2^{65}$. We also describe a new generic attack in the classical model for PRINCE.
\end{abstract}




\section{Introduction}

The multi-user setting is a very interesting practical scenario, which is sometimes overlooked in cryptography. Indeed, cryptosystems are designed to be used by many users, and usually cryptographers prove the security of their schemes in a single-user model except in some cases such as key exchange, public-key encryption and signatures. At EUROCRYPT 2012, Menezes [20] gave an invited talk pointing out the discrepancy between security proofs for message authentication code in the single-user and in the multi-user setting. As it was already been pointed out in 10, he showed that there is a straightforward reduction between the security proof for one user and the security proof for $L$ users with a success probability divided by $L$. Next, he recalled the key collision attack due to Biham [3] that matches this bound and that can be applied on various deterministic MACs (CMAC, SIV, OCB, EME, ... ). In this attack, the adversary asks the MAC tag of a single message $M$ for $L$ different users; we call this the set of secret MACs. Then, for a subset $W$ of size $N / L$ of known keys $(N$ is the key size), he computes $M A C(k, M)$ for all $k \in W$ and builds the set of public MACs. If a collision occurs between the public and secret sets, then we learn one of the $L$ secret keys 11 For MAC schemes with an 80-bit security level, it is possible with time/memory tradeoff to make this reasonably practical and derive a key recovery of a single key among a set of $L=2^{20}$ users, using time and memory $2^{40}$. Menezes thus insists that cryptographers have to consider this practical setting when devising or analyzing cryptosystems. For more results on multi-user attacks, the reader can also refer to [4].

In this paper, we are interested in collision-based attacks [24] in the multiuser setting. We rely on the distinguished point technique to propose new attacks on the generic discrete logarithm problem, on the Even-Mansour cipher and on PRINCE. Collision-based methods have been nicely improved by van Oorschot and Wiener to become parallelizable using the distinguished point technique of Rivest and Quisquater and Delescaille 22. Here, we extend these methods and apply them to cryptanalysis in the multi-user setting.

Our Contributions. From a cryptanalytic point of view, there are many ways to perform attacks in the multi-user setting. In this paper, we are interested by several scenarios. The first option is to recover all the users' keys (or a large fraction thereof) in time less than the product of the number of users by the time complexity to recover one key. Another direction is to improve Biham's attack and recover a single key in the multi-user setting with a reduced memory cost. Finally, we consider time/memory attacks starting with a precomputation whose result can then be used later to recover individual keys much faster.

Giant connected component. The multi-user setting for the discrete logarithm problem has been studied by Kuhn and Struik in [17]. They show that it is

${ }^{1}$ Provided that the tag length is greater than the key length. 
possible to adapt the parallel version of the Pollard rho technique with distinguished points to recover $L$ keys in time $\sqrt{N L}$ where $N$ is the size of the group as long as $L \ll \sqrt[4]{N}$. In the parallel version of Pollard rho method described by van Oorschot and Wiener, we run random walks in parallel, stop them once a distinguished point is reached and store this value for many starting points. We get a public set of distinguished points for the walks that begin at $y_{a}=g^{a}$ for which we know $a$ and a secret set from a user public key $y$ for starting points $y g^{b}$ where $b$ is known. Kuhn and Struik generalize this method by using many secret sets, one for each user. Once a distinguished point appears twice in the public and secret sets, the discrete logarithm of one user can be discovered, and consequently, we also know the discrete logarithm of all the distinguished points that were discovered during the random walks for this user. Therefore, as the number of "known" points increases, the probability of a collision between a secret point and a known one becomes higher. Similar results can be found in [19 1/2].

Here, we show another method that works without any restriction on $L$ and keeps the symmetry between all read points. Indeed, we do not have to wait until the first collision between a public point and a secret one happens, but we also consider collisions between secret points. More precisely, as soon as a collision between the public walks and the secret walks happens, we learn many discrete logarithms, since when two secret chains collide, we learn the difference between the discrete logarithm. We can then construct a graph whose vertices are the users and we add an edge if we know the difference of the discrete logarithm between these users. At some point, when the number of edges becomes slightly larger than the users, a giant component emerges in our random graph and if the public user is in this component (with high probability in time $2 L \ln L$ ), then the discrete logarithm of all users will be known.

Our method has an advantage towards the method proposed by Kuhn and Struik as we use parallelism extensively. However, a disadvantage is that in our case we do not learn any discrete logarithms until the very end, when a giant component appears in the graph. In contrast, Kuhn and Struik's algorithm is sequential and so they find each discrete logarithm one after the other. Overall, the main goal of section 2 is to provide an educational example of the graph connexity approach and show that it is much simpler to analyze.

Lambda Method for two different Even-Mansour style functions. We were also able to apply similar techniques on Even-Mansour with domain size $N$. Indeed, using some functions related to the encryption scheme, we show that we can learn the Xor between the keys of two users. The previous technique can also be used to recover the keys of all users. However, in this case, we get a new problem: the two functions we iterate are no longer the same. Consequently, contrary to the DL case, once a collision appears, the chains will no longer merge and we cannot use distinguished point technique. To solve this issue, we tweak the two functions and define related functions that will no longer merge but become parallel. We show that this parallel method is as efficient as the previous one. For instance, we show an attack that partially solves an open problem of Dunkelman et al. that asked to find a memoryless attack on Even-Mansour with $D$ queries to the 
secret function and $T=N / D$ to the public function with $D \ll \sqrt{N}$. We propose an attack that matches these bounds $\left(D=N^{2 / 5}, T=N^{3 / 5}\right)$ but where the memory is $N^{1 / 5}$ as an application of our lambda-method. Furthermore, we also describe a multi-user attack which allows to learn all the keys in a set of $N^{1 / 3}$ users in data complexity $N^{1 / 3+\epsilon}$ to each user and $T=N^{1 / 3+\epsilon}$ time complexity by combining the two algorithmic tools. This attack exhibits new tradeoff where the amortized data complexity per user times the time complexity is reduced to $N^{2 / 3+\epsilon}$ instead of $N$.

Application to PRINCE. PRINCE cipher [7 is a new block cipher recently introduced at ASIACRYPT 2012 with blocklength 64 bits and keylength 128 bits. Its design has a $\alpha$-reflection property which is a related-key relation that transforms the decryption algorithm to the encryption process with a relatedkey. Here, we propose generic attacks on the full number of rounds. At FSE 2014 [8, an attack on 10 rounds of PRINCE has been presented, with time complexity $2^{60.7}$ and data complexity $2^{57.94}$. In [15], an attack with slightly less than $2^{128}$ allows to break all the rounds, but our attacks have a particular low time complexity. They are similar to the one on Even-Mansour but we have to take into account that in PRINCE, the internal permutation uses a secret key. They make use both of the $\alpha$-reflection property and of the specific key scheduling of PRINCE, i.e. the relationship between the two whitening keys. The first attack allows to recover the keys of two users among a set of $2^{32}$ users in time $2^{65}$ and the second one allows to recover the keys of all users in time $2^{32}$ after a precomputation of time $2^{96}$ and $2^{64}$ in memory. Finally, we do not contradict the security bound showed in the original paper, but we show that different tradeoffs are possible.

Organization of the Paper. In section 2, we present our results on the discrete logarithm problem in the multi-user setting and we use the properties of random graph in this setting. Then, we present various results concerning the security of Even-Mansour: new time/memory/data (denoted T/M/D) tradeoffs, new time/memory (denoted T/M) attack solving the open problem of Dunkelman et al. and in the multi-user setting. In this part, we show how we can adapt the lambda-method when searching for collisions for two different functions based on the Even-Mansour idea. Finally, in the last section, we present various generic attacks on the PRINCE block cipher, one in the multi-user setting and the other in the classical model.

\section{Discrete Logarithms in the Multi-user Setting}

In this section, we present a new algorithmic idea for performing $\mathrm{T} / \mathrm{M}$ attacks with distinguished points in the multi-users setting. Our technique allows to compute the discrete logarithms of $L$ public keys $y_{i}=g^{x_{i}}$ for $i=1, \ldots, L$ in time $\widetilde{O}(\sqrt{N L})$ for any value of $L$ where $N=|\langle g\rangle|$. Starting from the parallel

${ }^{2}$ The $\widetilde{O}$ notation hides logarithmic terms. 
version of Pollard rho method [24, we compute $c L / 2$ chains consisting of pseudorandom walks from $y_{i}$ ( $c / 2$ chains for each user by randomizing the starting point) until we discover a distinguished point $d_{i} \in S_{0}$ where $S_{0}$ denotes the set of distinguished points 3 . Then, all distinguished points found are sorted and each collision between the distinguished points of different users $d_{i}$ and $d_{j}$ reveals a linear relation between $x_{i}$ and $x_{j}$. We also compute a few chains starting from random points for which the discrete logarithm is known $g^{x_{0}}$. Finally, we construct the random graph where the vertices are the public keys and we add an edge between $y_{i}$ and $y_{j}$ if we have a collision between $d_{i}$ and $d_{j}$ (this process can be described more formally using a random graph process). This edge is labelled with the linear relation between $x_{i}$ and $x_{j}$. Once we have computed a sufficient number of collisions, a small constant time the number of users, then a giant component will appear with high probability. More precisely, in a graph with $L$ vertices and $c L / 2$ randomly placed edges with $c>1$, there is a giant component whose size is almost exactly $(1-t(c)) L$, (see [6]) where:

$$
t(c)=\frac{1}{c} \sum_{k=1}^{\infty} \frac{k^{k-1}\left(c e^{-c}\right)^{k}}{k !} .
$$

For $c=4$, we get $1-t(c)=0.98$. The discrete logarithm of all the points in the component of the $x_{0}$ 's are known. If we want to recover the discrete logarithm of all users with overwhelming probability, we need $2 L \ln L$ edges to connect all connected components according to the coupon collectors problem and not $c L / 2$, as it is recalled in Theorem 2 .

Let $\ell$ the average length of the chains and $S_{0}$ the set of distinguished points. The average length of each chain is $\ell=N /\left|S_{0}\right|$. Assume we have computed $i$ chains that do not collide, the probability that the $(i+1)^{\text {th }}$ chain collides with one of the previous is $i \ell \times \ell / N$. Consequently, the expected number of collisions Coll is:

$$
\mathbb{E}[\text { Coll }]=\sum_{i=1}^{L-1} \frac{i \ell^{2}}{N} \approx \frac{L^{2}}{2} \cdot \frac{\ell^{2}}{N}=\frac{L^{2}}{2} \cdot \frac{\left(N /\left|S_{0}\right|\right)^{2}}{N}=\frac{L^{2} N}{2\left|S_{0}\right|^{2}} .
$$

We want the number of collisions to be larger than $c L / 2$, which implies $L^{2} N / 2\left|S_{0}\right|^{2} \geq c L / 2$, thus $\left|S_{0}\right| \leq \sqrt{L N / c}$. Consequently, the overall cost is dominated by the computation of the chains, i.e. $L \times N /\left|S_{0}\right|$ which is about $\sqrt{c L N}$ if $\left|S_{0}\right|=\sqrt{L N / c}$. Finally, in order to have $c L / 2$ edges in our graph, each user has to compute a small number of chains using a small number of random input points of the form $g^{x_{i}+r_{i}}$ for known value of $r_{i}$. The overall complexity of our attack is $\widetilde{O}(\sqrt{N L})$ for any value of $L$ while Kuhn and Struik analysis achieves the value $\sqrt{2 L N}$ for $L \ll \sqrt[4]{N}$.

Another possible approach to analyze known, unknown points and collisions between them would be to use a matrix. For this, we consider a symmetric matrix $M$ where $M[i, j]$ represents the linear relation between the discrete logarithms of $i$ and $j$. Then we apply a random variable in order to sparsify the matrix. More

3 This algorithm can also be adapted to the Pollard-lambda algorithm [21. 
precisely, we multiply the coefficient $(i, j)$ of the matrix by 1 with probability $p$ and by 0 with probability $(1-p)$, where these probabilities are independent. When we multiply by 1 , that means, that we know the differences between the discrete logarithms of $i$ and $j$.The question then becomes how many rows (with 2 non-zero coefficients) do we need to achieve full column-rank, which naturally leads to the same results: $O(L * \log (L))$. However, when considering rows with $O(\log (L))$ non-zero coefficients, we only needs $O(L)$ rows. This would imply that for multi-user discrete logarithms the overall complexity can be reduced by a factor $\log (L)$ to $O(\operatorname{sqr} t(L * N))$ by spending a factor $\log (L)$ more work in generating starting points of random combinations of $\log (L)$ known/unknown points (e.g., see [1]). We choose to analyze the complexity in the same form as Wiener and van Oorschot which is usually the case for crypto papers, i.e we do not care on the $\log N$ factors that arise in such birthday algorithms. Indeed, the Kuhn and Struik algorithm hides also a $\log (N)$ factor in order to get collisions with very high probability because a $1 / 2$ probability is not sufficient since we need many collisions of this type.

\section{Even-Mansour in the Single and Multi-user Settings}

\subsection{Brief Description of Even-Mansour}

At Asiacrypt 1991, Even and Mansour in [14 describe a very efficient design (called EM in the following) to construct a block cipher, i.e. a keyed permutation family $\Pi_{K_{1}, K_{2}}$ from a large permutation $\pi$. The key $K_{1}$ is first xored with the plaintext, then the fixed permutation is applied and finally the key $K_{2}$ is xored to obtain the final value.

$$
\Pi_{K_{1}, K_{2}}(P)=\pi\left(P \oplus K_{1}\right) \oplus K_{2} .
$$

Their main result is a security proof that any attack that uses $D$ on-line plaintext/ciphertext pairs (queries to $\Pi$ ) and $T$ off-line computations (queries to $\pi$ ) must satisfy $D T=N$, where $N=2^{n}$ with $n$ the size of the plaintext and key and which will be called the EM curve. The important part of the proof is that it is a lower bound for all attacks including known-plaintext attacks. It appears that the use of two keys $K_{1}$ and $K_{2}$ does not add much more resistance to the scheme. This variant of using $K=K_{1}=K_{2}$ has been proposed under the name Single-Key Even-Mansour and we denote it by $\Pi_{K}$. The security of this minimal version has been proved secure with the same bound as for the two-key version by Dunkelman et al. This minimal version is amazingly resistant and guarantees the same security bound, but it is not unexpected since usually the attacks look for the two keys independently and once the key $K_{1}$ is recovered, there is no security for $K_{2}$. In the following, we see that the two-key version does not improve the security since most of the attacks on the single-key can be levered to this version.

In this section, we describe new results concerning the security of the EvenMansour scheme which has recently been the subject of many papers [1318]. 
We recall the basic attacks and then, we present a basic $\mathrm{T} / \mathrm{M}$ tradeoff for known plaintext attacks with better on-line complexity (Sect. 3.3) and a better T/M tradeoff for adaptive queries (Sect. 3.4). For this attack, we introduce our second algorithmic trick to discover collisions for two different functions based on the Even-Mansour construction. The main difficulty we have to solve is that when a lambda-like method is used to recover collisions, if two different functions are used, after the collision, the chain will no longer merge. To this end, we adapt the lambda-method to have parallel chains when the collision happens. Finally, we show that in the multi-user setting (Sect. 3.5) the precomputation cost can be amortized. It is possible to balance all the complexities to recover all the keys of $N^{1 / 3}$ users with $N^{1 / 3+\epsilon}$ adaptive queries to each user, a precomputation time of $N^{1 / 3+\epsilon}$ and the attack requires $N^{1 / 3+\epsilon}$ in memory and $N^{1 / 3+\epsilon}$ for the on-line time.

\subsection{Previous Attacks on Even-Mansour}

In [12, Daemen showed that the EM curve $T D=N$, is valid for a known plaintext attack at the point $(T=N / 2, D=2)$. He also gave a chosen-plaintext attack that matches the EM curve for any value of $D$ and $T$ and in particular at the point $\left(T=N^{1 / 2}, D=N^{1 / 2}\right)$. Later, Biryukov and Wagner described a sliding attack that matches the EM curve for known-plaintext but only at the point $\left(T=N^{1 / 2}, D=N^{1 / 2}\right)$. Recently, Dunkelman et al. introduce a new twist on the sliding attack whose complexities match the whole curve for any value of $D$ and $T$ using a known-plaintext attack which is exactly the result proved by Even and Mansour. Finally, Dunkelman et al. also provide a slidex attack on the two-key Even-Mansour scheme.

Simpler collision-based attack on the Single-Key Even-Mansour. In the singlekey case a simpler attack achieves the same performance. The basic idea is to apply the Davies-Meyer construction to $\Pi$ and to $\pi$. More precisely, write:

$$
F_{\Pi}(x)=\Pi(x) \oplus x \quad \text { and } F_{\pi}(x)=\pi(x) \oplus x .
$$

For any value of $x$, the equality $F_{\Pi}(x)=F_{\pi}(x \oplus K)$ is satisfied. Moreover, any collision between these two functions $F_{\Pi}(x)=F_{\pi}(y)$ indicates that $x \oplus y$ is a likely candidate for the key $K$.

With this idea in mind, the problem of attacking the single key Even-Mansour scheme is reduced to the problem of finding a collision (or rather a few collisions) between $F_{\Pi}$ and $F_{\pi}$. The simplest approach is simply to compute $F_{\pi}$ on $T$ distinct random values and $F_{\Pi}$ on $D$ distinct random values. When $D T \approx N$, one expects to find the required collisions.

Moreover, this can be done in a more efficient way by using classical collision search algorithms with reduced memory. Indeed, it is possible to use Floyd's cycle finding algorithm to obtain such a solution for the special case $D=T=N^{1 / 2}$, without using memory. However, in this case the attack is no longer a knownplaintext attack and becomes an adaptively chosen plaintext attack. 
Dunkelman, Keller and Shamir ask whether it is possible to generalize this and to find memoryless attacks using $D$ queries to $\Pi$ and $N / D$ to $\pi$ where $D \ll N^{1 / 2}$ ?

In this paper, we partially answer this question, proposing attacks that use less than $D \ll N^{1 / 2}$ data and memory lower than $\min (T, D)$ if we require the unkeyed queries to be precomputed. Without this requiring, we achieve a memoryless attack.

\subsection{Extending the Simple Attack}

Dealing with two keys Even-Mansour. A first important remark is that the simple attack on Single-Key EM can be extended to the two-key case. The idea is simply to replace the function $\pi(x) \oplus x$ by another function with similar properties. A first requirement is that the chosen function needs to be expressed by two different formulas, one based on $\pi$ and the other on $\Pi$. The other requirement is that a collision on two evaluations, one of each type, should yield good candidates for the keys.

We now construct the required function and show that the simple attack on the single-key variant can be extended to two keys. We first choose a random non-zero constant $\delta$ and let:

$$
F_{\Pi}(x)=\Pi(x) \oplus \Pi(x \oplus \delta) \text { and } F_{\pi}(x)=\pi(x) \oplus \pi(x \oplus \delta) .
$$

We remark that $F_{\Pi}(x)=F_{\pi}\left(x \oplus K_{1}\right)$ and that $F_{\Pi}(x \oplus \delta)=F_{\pi}\left(x \oplus K_{1}\right)$ are both satisfied. As a consequence, every collision now suggests two distinct input keys $K_{1}=x \oplus y$ and $K_{1}=x \oplus y \oplus \delta$. Except for this detail, the attack remains unchanged. Note that once $K_{1}$ has been found, recovering $K_{2}$ is a trivial matter.

Reducing the on-line time complexity. In this section, we focus on known-plaintext attacks and we first show that the EM security model does not separate the online and off-line time complexities, as usually done in $\mathrm{T} / \mathrm{M} / \mathrm{D}$ tradeoff. It is then possible to use T/M/D tradeoff for this blockcipher design as suggested in [5] by Biryukov and Shamir.

Let us separate the on-line time denoted by $T_{\text {on }}$ and the off-line time denoted by $T_{\text {off }}$. Clearly, the total time complexity $T$ is $T_{\text {on }}+T_{\text {off }}$.

The main idea of this section is to use a different approach to find a collision between $F_{\Pi}$ and $F_{\pi}$. More precisely, given a value of $F_{\Pi}$, we try to invert $F_{\pi}$ on this value. If we succeed, we clearly obtain the desired collision. In order to inverse $F_{\pi}$, we rely on Hellman's algorithm. The T/M/D tradeoff is

$$
T_{\text {on }} M^{2} D^{2}=N^{2} \text { and } D^{2} \leq T_{\text {on }} \leq N \text {. }
$$

In order to fully use Hellman tradeoff with multiple tables, we can use the $\delta$ in the definition of the function $F_{\pi}(x)=\pi(x) \oplus \pi(x \oplus \delta)$ to define different and independent functions for each table. These attacks achieve $T_{\text {on }} D \ll N$ while $T D=N$. 
Using less data than memory. Despite its optimal efficiency in term of knownplaintext attack matching the EM curve, the Slidex attack presents an important drawback. Indeed, the public permutation $\pi$ needs to be evaluated at points which depend on the result of the queries to the keyed Even-Mansour construction $\Pi$. As a consequence, with this attack, it is not possible to precompute the queries to $\pi$ in order to improve the online time required to obtain the key to $\Pi$.

Our previous attack based on Hellman's tables no longer requires adaptive queries, however, it is less costly than the Slidex attack in term of on-line time complexity but more costly than the simple collision-based attack (which uses adaptive chosen plaintext). The goal of the next subsection is to present an attack on $\Pi$, which is based on classical collision search algorithms and works by using queries to $\pi$ and $\Pi$ without any cross-dependencies. However, the queries to $\Pi$ are adaptive but this new attack is more flexible to perform $\mathrm{T} / \mathrm{M}$ tradeoff.

\subsection{Time/Memory/Data Tradeoff Attack on Even-Mansour}

Attacking Even-Mansour using distinguished points methods. In order to attack Even-Mansour using a distinguished point method, we would like to construct a set of chains using the public permutation $\pi$ and then find a collision with a chain obtained from the keyed permutation $\Pi$. One difficulty is that chains computing from $\pi$ and from $\Pi$ can never merge since they are based on different functions contrary to discrete logarithm section. We introduce here a new idea to solve this dilemma when the functions are based on the Even-Mansour construction. Let us define:

$$
F_{\Pi}(x)=x \oplus \Pi(x) \oplus \Pi(x \oplus \delta) \text { and } F_{\pi}(x)=x \oplus \pi(x) \oplus \pi(x \oplus \delta) .
$$

We remark that $F_{\Pi}\left(x \oplus K_{1}\right)=F_{\pi}(x) \oplus K_{1}$. As a consequence, two chains based on $F_{\Pi}$ and $F_{\pi}$ cannot merge, but they may become parallel. Indeed, using the equation $F_{\Pi}\left(x \oplus K_{1}\right)=F_{\pi}(x) \oplus K_{1}$ and let two points $X$ and $x$ such that $X=$ $x \oplus K_{1}$, where $X$ (resp. $x$ ) belongs to an $F_{\Pi}$ chain (resp. $x$ belongs to an $F_{\pi}$ chain), the next element $Y=F_{\Pi}(X)$ in the $F_{\Pi}$ chain and the next element $y=F_{\pi}(x)$ in the $F_{\pi}$ chain will satisfy:

$$
Y=F_{\Pi}(X)=F_{\Pi}\left(x \oplus K_{1}\right)=F_{\pi}(x) \oplus K_{1}=y \oplus K_{1} .
$$

So $Y=y \oplus K_{1}$, which means that $Y$ and $y$ satisfy the same relation as $X$ and $x$, and so on. Therefore, as soon as by chance $X=x \oplus K_{1}$ where $X$ is an element of an $F_{\Pi}$ chain and $x$ is an element of an $F_{\pi}$ chain, the same relation remains with the subsequent points of the two chains, i.e. we get two parallel chains.

Moreover, the detection of this good event is compatible with the distinguished point method. Indeed, it suffices to define a distinguished point $x$ as a point with a value of $\pi(x) \oplus \pi(x \oplus \delta)$ in $S_{0}$. Similarly, for chains constructed by using $F_{\Pi}$, we define a distinguished point $X$ as a point with a value of $\Pi(X) \oplus \Pi(X \oplus \delta)$ in $S_{0}$. Now if $X=x \oplus K_{1}$ and $x$ is a distinguished point in a $\pi$ chain, then since

$$
\Pi(X) \oplus \Pi(X \oplus \delta)=\pi\left(X \oplus K_{1}\right) \oplus \pi\left(X \oplus K_{1} \oplus \delta\right)=\pi(x) \oplus \pi(x \oplus \delta),
$$


the point $X$ is also a distinguished point in the $\Pi$ chain, and therefore $X \oplus x$ gives a candidate for $K_{1}$. Since the values $\pi(x) \oplus \pi(x \oplus \delta)$ and $\Pi(X) \oplus \Pi(X \oplus \delta)$ are needed to compute the next element in the chains, using this definition does not add any extra cost for distinguished point detection. The important point, is that for a parallel chain based on $F_{\Pi}$, a point $X=x \oplus K_{1}$ corresponds to a distinguished point $x$ if and only if $\Pi(X) \oplus \Pi(X \oplus \delta)$ is in $S_{0}$.

An important difference compared to the classical search for collisions is that we do not need to backtrack to the beginning of the chains and identify where the chains merge. Indeed, seeing parallel distinguished points suffices to get candidates values for $K_{1}$.

Analysis of the attack with precomputation. Since there is a clear symmetry between the keyed and unkeyed queries, we may assume that the number of unkeyed queries $T$ is larger than the number of keyed queries $D$. Let $B_{T}$ the number of unkeyed chains to increase the probability of a collision between keyed and unkeyed chains. Moreover, this is the most reasonable scenario, since keyed queries are usually the most constrained resource. In this case, we need to choose the expected length $\ell$ of the chains we are going to construct and $B_{T}$ that satisfy the following relations:

$$
T=\ell \cdot B_{T} \quad \text { and } \quad N=B_{T} \ell^{2} .
$$

Thus, $\ell=N / T$ and $B_{T}=T^{2} / N$. The required memory to store those chains is of size $O\left(B_{T}\right)$.

After terminating the computation of the unkeyed chains, we can turn to the keyed side. On this side, we want to perform about $D=N / T$ evaluations of the function. Since $D=\ell$, this means that we compute a single keyed chain and expect it to (parallel) collide with an unkeyed chain.

We are interested in values for $M$ such that $M<D$. Consequently, as $M=$ $T / D=N / D^{2}$, we have $N<D^{3}$. Let us consider $N^{1 / 3}<D=N^{\alpha}<N^{1 / 2}$. For example, if $D=N^{2 / 5}$ and $T=N^{3 / 5}$, then $M=N^{1 / 5}$ is much smaller than $N^{2 / 5}$. This attack requires a number of data $D \ll N^{1 / 2}$ and despite this attack is not memoryless (as in the open problem), the memory is less than the data.

Relaxing the precomputation requirement. Another alternativet is to perform the same attack while computing the keyed queries before the unkeyed ones. In this case, since there is a single keyed chain to be stored, we can achieve the attack using a constant amount of memory. Moreover, this variation works for any $D=N^{\alpha} \leq N^{1 / 2}$ using $T=N / D$.

\subsection{Attacks in the Multi-user Setting}

In the multi-user setting, we assume that $L$ different users are all using the Even-Mansour scheme based on the same public permutation $\pi$, with each user

\footnotetext{
${ }^{4}$ We thank an anonymous reviewer of Asiacrypt 2014 for pointing this out.
} 
having its own key 5 , chosen uniformly at random and independently from the keys of the other users.

Of course, the attack from Section 3.4 can be easily applied in this context. Depending on the exact goal of the cryptanalysis, we have two main options:

1. If the goal is to recover the key of all users, the previous attack can be applied by repeating the $D$ key-dependent queries for each user, while amortizing the $T$ unkeyed queries across users. A typical case is to consider $L=N^{1 / 3}$ users, to perform $T=N^{2 / 3+\epsilon}$ unkeyed queries $\left(N^{1 / 3+\epsilon}\right.$ chains of $N^{1 / 3}$ queries, memory $\left.N^{1 / 3}\right)$. For each new user, we need $N^{1 / 3+\epsilon}$ key-dependent queries. As a consequence, the amortized cost per user (up to constant factors $c_{0}=20$ ) is $N^{1 / 3+\epsilon}$ queries of each type and the required memory also is $N^{1 / 3}$.

2. If the goal of the cryptanalyst is to obtain at least one user key among all the users, it suffices to split the $D$ key-dependent queries arbitrarily across the users.

However, we present in this section a much more efficient tradeoff in the multiuser setting. This tradeoff becomes possible without precomputation in $N^{2 / 3}$, but by distributing the unkeyed queries among the users and by reusing the graph algorithmic idea of the section 2 For this, we construct a graph whose vertices are labelled by the users. Whenever we obtain a collision $F_{\Pi}{ }^{(i)}(x)=F_{\Pi}{ }^{(j)}(y)$ for users $i$ and $j$, we add an edge between the corresponding vertices labelled with $x \oplus y$ which is expected equal to $K^{(i)} \oplus K^{(j)}$. Note that this indicates that we know the exclusive-or of the first keys of the two users.

If we have $L$ vertices and $c L / 2$ randomly edges with $c=4$, there is a giant component whose size is $98 \%$ of the points, and with $c L \ln L$, all the points are in this component with overwhelming probability. Consequently, we obtain the exclusive-or of the first keys for an arbitrary pair of users. To conclude the attack, it suffices to find a single collision between any of the users functions $F_{\Pi}$ of the large connected component and the unkeyed function $F_{\pi}$ to reveal all the keys of these users.

\section{Algorithm Description.}

1. Create a constant number $c / 2$ of chains for each user up to a distinguished point.

2. Sort the distinguished points.

3. Bring together the distinguished points into subsets, where we test whether the key candidate is really the good one. It is indeed easy to check with a few more queries if the xor of two keys is correct.

4. Construct the giant component and expect that the public user (the user with the unkeyed function), lies in this giant component. To this end, we initially begin with the set of reachable users containing only the public user. Then, we add to this set all the users that are in a group where a reachable user is present. At some point, the reachable set is stable and we stop.

\footnotetext{
${ }^{5}$ Or key-pair depending on whether we are considering the single or dual key scheme.
} 
5. From the public user, we cross over the giant component and determine the keys of each user.

The first step requires $c L \ell / 2$ data and time $O(c \ell)$ on average per user where $\ell$ is the average length of the chains. Then, the remaining steps are performed in time linear in the number of users $L$. Typical parameters are: for an arbitrary small positive constant $c$, we expect with $N^{1 / 3}$ users, $c \cdot N^{1 / 3}$ queries per user and $N^{1 / 3}$ unkeyed queries, to recover almost all the $N^{1 / 3}$ keys with overwhelming probability. If we want to recover all users, we need to have $L \ln L=c N^{1 / 3} \ln N=N^{1 / 3+\epsilon}$ edges (instead of $c L / 2$ ) to connect all components according to the coupon collector's problem.

Analysis of the attack. We want to use results from graph theory to prove the correctness of our algorithm, this means that we have to prove that the assumptions of the giant component theorem are satisfied. We have to show that we construct of a random graph according to the Erdös-Rényi model of random graphs, in which each possible edge connecting pairs of a given set of $L$ vertices is present, independently of the other edges, with probability $p$. In this case, we know that with this model of random graph, if the number of edges $c . L / 2$ is larger than the number of vertices $L$, there is with high probability a single giant component, with all other components having size $O(\log L)$ according to [6].

Consequently, we need to prove that we construct a random graph and that the edges are added independently of each others. We will define an idealized version of the attack and we will show that the attack works in this version. Then, we will prove that the idealized version and the attack are equivalent using simulation argument.

In the idealized model, the simulator randomly chooses $L$ keys $K_{1}, \ldots, K_{L}$ uniformly at random. Then it iterates the functions $F_{\Pi}^{(i)}(x)=K_{i} \oplus F_{\pi}\left(x \oplus K_{i}\right)$ until $x_{\ell} \oplus K_{i} \in S_{0}$, where $S_{0}$ is the set of pairs containing a distinguished point $d_{i}$ and an identificator of this point $i d\left(d_{i}\right)$. The identificators are unique, which means that we do not have collision on them. Finally, the simulator reveals the identificator of the point $x_{\ell} \oplus K_{i}$ and the point $x_{\ell}$. The value $K_{i}$ cannot be recovered from the information that the simulator returns.

To show that the attack works in this ideal model, we just have to see that if two users have the same identificator, then $x_{\ell} \oplus K_{i}=x_{\ell^{\prime}} \oplus K_{j}$ and therefore $x_{\ell} \oplus x_{\ell^{\prime}}=K_{i} \oplus K_{j}$ which is the same information as in the real attack.

Now, we will prove that the simulator does not need to know $F_{\Pi \Pi}^{(i)}$ and can simulate the information by only using the public random function $F_{\pi}$ and that the distribution of its outputs is indistinguishable from the idealized model. The simulator generates at random $L$ random keys for the EM scheme. For each key, we will show that the pairs distinguished point/identificator can be generated only using $F_{\pi}$. Indeed, $x_{\ell}$ the $\ell$ th iteration of $F_{\Pi}^{(i)}$ with key $K_{i}$ from the value $x_{0}$ is the value $K_{i} \oplus x_{\ell}$ and this value is also the result of the iteration of the public function $F_{\pi}$ from the value $x_{0} \oplus K_{i}$. Consequently, to generate the pairs (distinguished point, identificator), the simulator can compute $\left(x_{\ell} \oplus K_{i}, i d\left(x_{\ell}\right)\right)$ without interacting with the users. As in this last case, the pairs are generated 
at random without interacting and knowing the function and since the function $F_{\pi}$ are random, the edges in the graph are added at random and independently of each others and so that the graph is a random graph according to the ErdösRényi graph model.

Experimental results. We implement the previous attacks on an Even-Mansour cryptosystem using the DES with a fixed key and $n=64$. We simulate $2^{22}$ users and for each user we create 8 chains ( 80 for the public user). We use distinguished points containing 21 zeroes and so the expected length is $2^{21}$ on average. We bound the length of the chains to $2^{24}$, this means that if we remove the chain if we have not seen a distinguished point after $2^{24}$ evaluations. In all, we have generated $33,543,077$ chains $\left(2^{25}=33,554,432\right.$, it misses the abandoned chains) and the number of groups containing at least two parallel chains is $4,109,961$. Experimentally, the size of the giant component contains 3, 788, 059 users (among the $4,194,304)$ and so we can deduce the keys of $90 \%$ of the users. This result is what is expected from theory since the number of vertices in this experiment is below the number of nodes. The $98 \%$ that is previously given as result in section 3.5. would require twice as many vertices.

The time to generate the chains is $1600 \mathrm{sec}$ using 4096 cores in parallel and the analysis of the graph requires a few minutes on a standard PC.

\section{Attacks on the PRINCE Cipher in the Multi-user and Classical Setting}

PRINCE is a lightweight block cipher published at ASIACRYPT 2012 [7]. It is based on the $F X$ construction [16] which is actually an Even-Mansour like construction. PRINCE has been the interest of many cryptanalysts 92315$]$ who attack either the full cipher, or its reduced version.

The designers of PRINCE claim that its security is ensured up to $2^{127-n}$ operations when an adversary acquires $2^{n}$ plaintext/ciphertext pairs. This bound has been reduced in [15] to $2^{126}$ operations with a single plaintext/ciphertext pair. After a brief presentation of PRINCE, we describe a generic attack in the multi-user setting that allow to recover the key of a pair of users in a set of $2^{32}$ users with complexity $2^{64}$ computations. The identification of the pair of users uses the idea similar to the attack on Even-Mansour. However, details are different since PRINCE is not an Even-Mansour scheme as the internal permutation uses a secret key. Finally, we present another generic attack in the classical model that after a precomputation of $2^{96}$ time and $2^{64}$ in memory, allows to recover the key of every single user in time $2^{32}$. Both attacks work for all rounds of PRINCE.

\subsection{Brief Description of PRINCE}

PRINCE [7] uses a 64-bit block and a 128-bit key which is split into two equal parts of 64 bits, i.e. $k=k_{0} \| k_{1}$. In order to extend the key to 192 bits it uses 
the mapping $k=\left(k_{0} \| k_{1}\right) \rightarrow\left(k_{0}\left\|k_{0}^{\prime}\right\| k_{1}\right)$ where $k_{0}^{\prime}$ is derived from $k_{0}$ by using a linear function $L^{\prime}$ :

$$
L^{\prime}\left(k_{0}\right)=\left(k_{0} \ggg 1\right) \oplus\left(k_{0} \gg 63\right),
$$

where $\gg$ denotes the right shift and $\gg$ the rotation of a 64 -bit word. While subkeys $k_{0}$ and $k_{0}^{\prime}$ are used as input and output whitening keys, the 64-bit key $k_{1}$ is used for the 12-round internal block cipher which is called PRINCE $\mathrm{P}_{\text {core }}$. For simplicity, we refer to it as the core of PRINCE or simply the core function and we denote it by Pcore. So every plaintext $P$ is transformed into the corresponding ciphertext $C$ by using the function $E_{k}(P)=k_{0}^{\prime} \oplus$ Pcore $_{k_{1}}\left(P \oplus k_{0}\right)$ where Pcore uses the key $k_{1}$ (see Fig 1).

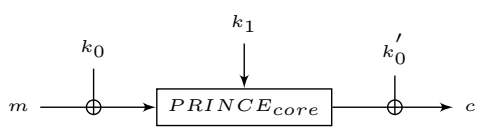

Fig. 1. Structure of PRINCE

The core function consists of a key $k_{1}$ addition, a round constant $\left(R C_{0}\right)$ addition, five forward rounds, a middle round, five backward rounds and finally a round constant $\left(R C_{11}\right)$ and a key $k_{1}$ addition. The full schedule of the core is shown in Fig. 2

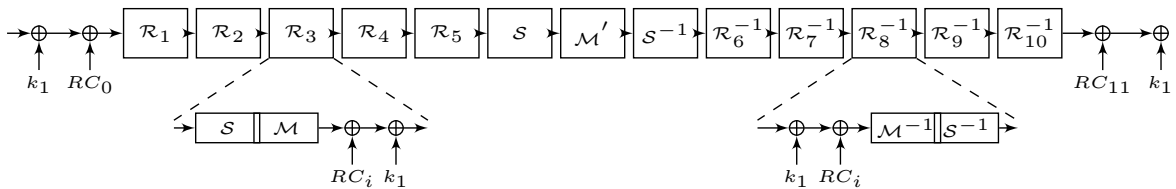

Fig. 2. Structure of the core of PRINCE

Each forward round of the core is composed by a 4-bit Sbox layer $(S)$, a linear layer $(64 \times 64$ matrix $M)$, an addition of a round constant $R C_{i}$ for $i \in\{1, \ldots, 5\}$, and the addition of the key $k_{1}$. The linear $M$ layer is defined as $M=S R \circ M^{\prime}$ where $S R$ is the following permutation

\section{\begin{tabular}{|l|l|l|l|l|l|l|l|l|l|l|l|l|l|l|l|l|l|l|l|l|l|l|l|l|l|l|l|l|l|}
\hline 0 & 1 & 2 & 3 & 4 & 5 & 6 & 7 & 8 & 9 & 10 & 11 & 12 & 13 & 14 & 15
\end{tabular}$\rightarrow$\begin{tabular}{ll}
0 \\
\hline
\end{tabular}}

The $M^{\prime}$ layer, which is only used in the middle rounds, can be seen as a mirror in the middle of the core as the 5 backward rounds are defined as the inverse of the 5 forward rounds.

In every $R C_{i}$-add step, a 64-bit round constant is XORed with the state.

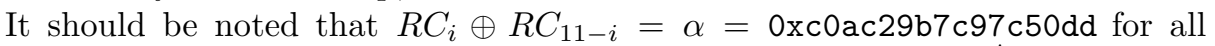
$0 \leq i \leq 11$. From this, but also from the fact that the matrix $M^{\prime}$ is an involution, we can perform the decryption function of PRINCE by simply performing the encryption procedure with inverse order of keys $k_{0}$ and $k_{0}^{\prime}$ and by using the 
key $k_{1} \oplus \alpha$ instead of $k_{1}$. That means, that for any key $\left(k_{0}\left\|k_{0}^{\prime}\right\| k_{1}\right)$, we have $D_{\left(k_{0}\left\|k_{0}^{\prime}\right\| k_{1}\right)}(\cdot)=E_{\left(k_{0}^{\prime}\left\|k_{0}\right\| k_{1} \oplus \alpha\right)}(\cdot)$. This property is called the $\alpha$-reflection property of PRINCE.

\subsection{Attack on PRINCE in the Multi-user Setting}

In the multi-user setting, we assume that we have $L$ different users which are all using the block cipher PRINCE. Each user $U_{i}$ with $0 \leq i<L$, chooses her key $k^{(i)}=k_{0}^{(i)} \| k_{1}^{(i)}$ at random and independently from all the other users. In order to attack PRINCE using the distinguished point method, we first construct a set of chains for every user using the function of PRINCE. For this, we use the function defined as follows:

$$
F_{k_{0}^{(i)}, k_{0}^{(i)}, k_{1}^{(i)}}(x)=x \oplus \operatorname{PRINCE}_{k_{0}^{(i)}, k_{0}^{\prime(i)}, k_{1}^{(i)}}(x) \oplus \operatorname{PRINCE}_{k_{0}^{(i)}, k_{0}^{\prime(i)}, k_{1}^{(i)}}(x \oplus \delta)
$$

where $\delta$ is an arbitrary but fixed non zero constant. The key $k_{0}^{\prime}(i)$ vanishes from the equation and the function $F$ thus takes the following form:

$$
F_{k_{1}^{(i)}}(x)=x \oplus \text { Pcore }_{k_{1}^{(i)}}\left(x \oplus k_{0}^{(i)}\right) \oplus \text { Pcore }_{k_{1}^{(i)}}\left(x \oplus k_{0}^{(i)} \oplus \delta\right) .
$$

For every user $U_{i}$, we create one encryption $(\mathcal{E})$ chain and one decryption $(\mathcal{D})$ chain which are both based on the function $F$ defined above. $\mathcal{E}$ uses the encryption function of PRINCE whereas $\mathcal{D}$ uses the decryption function. And so, for the user $U_{i}$, we define functions $\mathcal{E}$ and $\mathcal{D}$ as follows:

$$
\begin{aligned}
& \mathcal{E}_{k_{0}^{(i)}, k_{1}^{(i)}}\left(x_{j}^{(i)}\right)=x_{j+1}^{(i)}=x_{j}^{(i)} \oplus \operatorname{Pcore}_{k_{1}^{(i)}}\left(x_{j}^{(i)} \oplus k_{0}^{(i)}\right) \oplus \operatorname{Pcore}_{k_{1}^{(i)}}\left(x_{j}^{(i)} \oplus k_{0}^{(i)} \oplus \delta\right) \\
& \mathcal{D}_{k_{0}^{\prime(i)}, k_{1}^{(i)} \oplus \alpha}\left(y_{j}^{(i)}\right)=y_{j+1}^{(i)} \\
&=y_{j}^{(i)} \oplus \operatorname{Pcore}_{k_{1}^{(i)} \oplus \alpha}\left(y_{j}^{(i)} \oplus k_{0}^{\prime(i)}\right) \\
& \oplus \operatorname{Pcore}_{k_{1}^{(i)} \oplus \alpha}\left(y_{j}^{(i)} \oplus k_{0}^{(i)} \oplus \delta\right) .
\end{aligned}
$$

Let us define:

$$
\begin{aligned}
& f^{\mathcal{E}}=\operatorname{Pcore}_{k_{1}^{(i)}}\left(x_{j}^{(i)} \oplus k_{0}^{(i)}\right) \oplus \operatorname{Pcore}_{k_{1}^{(i)}}\left(x_{j}^{(i)} \oplus k_{0}^{(i)} \oplus \delta\right) \text { and } \\
& f^{\mathcal{D}}=\operatorname{Pcore}_{k_{1}^{(i)} \oplus \alpha}\left(y_{j}^{(i)} \oplus k_{0}^{\prime(i)}\right) \oplus \operatorname{Pcore}_{k_{1}^{(i)} \oplus \alpha}\left(y_{j}^{(i)} \oplus k_{0}^{\prime(i)} \oplus \delta\right) .
\end{aligned}
$$

We create encryption chains until $f^{\mathcal{E}}$ reaches a distinguished point (resp. decryption chains until $f^{\mathcal{D}}$ reaches a distinguished point). We search for a collision between the encryption and the decryption chain.

Let us consider two users, $U_{1}$ and $U_{2}$. Whenever the chains $\mathcal{E}_{k_{0}^{(1)}, k_{1}^{(1)}}\left(x^{(1)}\right)$ and $\mathcal{D}_{k_{0}^{(2)}, k_{1}^{(2)} \oplus \alpha}\left(y^{(2)}\right)$ arrive at the same distinguished point, we suspect that these 
two chains have become parallel. As the core of PRINCE is only parametrized by the key $k_{1}$, when we arrive at the same distinguished point we obtain a probable collision between keys $k_{1}^{(1)}$ and $k_{1}^{(2)} \oplus \alpha$ used in Pcore. However, we must verify that this is a real collision and not just a random incident. For this, we verify that next points of $f^{\mathcal{E}}$ and $f^{\mathcal{D}}$ after reaching a distinguished point, continue to remain equal. If we obtained a real collision we know that:

$$
k_{1}^{(1)}=k_{1}^{(2)} \oplus \alpha .
$$

This indicates that $x^{(1)} \oplus y^{(2)}$ is expected equal to $k_{0}^{(1)} \oplus k_{0}^{\prime(2)}$. It is obvious that since $k_{1}^{(1)}=k_{1}^{(2)} \oplus \alpha$ we will also have $k_{1}^{(1)} \oplus \alpha=k_{1}^{(2)}$. This indicates that we also know $k_{0}^{\prime(1)} \oplus k_{0}^{(2)}$.

Thus, we have:

$$
k_{0}^{(1)} \oplus k_{0}^{\prime(2)}=A \text { and } k_{0}^{\prime(1)} \oplus k_{0}^{(2)}=B \quad(*) .
$$

Let $\left\{a_{63}, \ldots, a_{0}\right\}$ be the representation of the bits of $k_{0}^{(1)}$ and $\left\{b_{63}, \ldots, b_{0}\right\}$ the representation of bits of $k_{0}^{(2)}$. As, from the definition of PRINCE, $k_{0}^{\prime}=\left(k_{0} \ggg\right.$ 1) $\oplus\left(k_{0} \gg 63\right)$, we have that:

$$
k_{0}^{\prime(1)}=\left\{a_{0}, a_{63}, \ldots, a_{2}, a_{1} \oplus a_{63}\right\} \text { and } k_{0}^{\prime(2)}=\left\{b_{0}, b_{63}, \ldots, b_{2}, b_{1} \oplus b_{63}\right\} .
$$

From $(*)$, we construct the system:

$$
\begin{aligned}
\left\{a_{63}, \ldots, a_{0}\right\} \oplus\left\{b_{0}, b_{63}, \ldots, b_{2}, b_{1} \oplus b_{63}\right\} & =\left\{A_{63}, \ldots, A_{0}\right\} \\
\left\{b_{63}, \ldots, b_{0}\right\} \oplus\left\{a_{0}, a_{63}, \ldots, a_{2}, a_{1} \oplus a_{63}\right\} & =\left\{B_{63}, \ldots, B_{0}\right\}
\end{aligned}
$$

As this is an inversible linear system, we can easily find $k_{0}^{(1)}$ and $k_{0}^{(2)}$. Note that once $k_{0}^{(i)}$ has been found, recovering $k_{1}^{(i)}$ can be done with an exhaustive search whose cost is $2^{64}$.

Analysis of the attack. Once the computation of a chain is finished we have to store $\left(x_{\ell-1}, d, d+1\right)$ where $d$ is the distinguished point, $x_{\ell-1}$ is the point before the chain reaches a distinguished point and $d+1$ is the point after the chain reached a distinguished point. We need to store $x_{\ell-1}$ as we have to test if the found collision is useful and we also need to store $d+1$ to test if it is a real collision. If not, the search must continue.

As mentioned, PRINCE uses a 128-bit key which is split into two 64-bit parts, i.e. $k=k_{0} \| k_{1}$. The attack consists in identifying and recovering all key material of a pair of users $i$ and $j$ for whom $k_{1}^{(i)}=k_{1}^{(j)} \oplus \alpha$. We expect to find a collision $k_{1}^{(i)}=k_{1}^{(j)} \oplus \alpha$ between two different users with high probability when the number of users will be at least $2^{32}$. So the attack uses a set of $2^{32}$ users and for each one we create 2 chains (encryption and decryption chain). The cost per user is $2^{32}$ operations and the total cost for recovering the keys $k_{0}$ of 2 users is approximately $2^{64}$ operations. For recovering $k_{1}$, the cost of the exhaustive search is $2^{64}$. So in total, we can deduce both $k_{0}$ and $k_{1}$ in $2^{65}$ operations. 


\subsection{Attack in the Classical Model}

We show in this section that a classical attack that also uses the distinguished points technique can also be possible. For this, we will create encryption chains from the function $\mathcal{E}$ defined in section 4.2 .

Precalculation. In the first phase of the attack, we aim to create encryption chains for every possible key $k_{1}^{(i)}$ with $0 \leq i<2^{64}$. More specifically, for every possible $k_{1}^{(i)}$, we set $k_{0}^{(i)}=0$ and we create for every $(i)$ a chain $\mathcal{S}_{i}$ from the function $\mathcal{E}$ with length $2^{32}$. We store all chains $\mathcal{S}_{i}$.

Attack. Now, our purpose is to find a collision with one of the chains created with the zero key $k_{0}^{(i)}$. For this, for a random starting point $x_{0}$ and for keys $k_{0}$ and $k_{1}$ we will calculate an encryption chain $\mathcal{T}$ from the function $\mathcal{E}$. The chain $\mathcal{T}$ will collide with high probability with one of the chains $\mathcal{S}_{i}$. As described in previous section 4.2, when we detect a collision between two distinguished points, we know that the chains had become parallel and so we obtain $k_{0}^{(i)} \oplus k_{0}$. As the key $k_{0}^{(i)}=0$, we finally obtain the unknown $k_{0}$.

Analysis of the attack. For the precalculation phase, for every $2^{64}$ possible keys we calculate a chain with length $2^{32}$ and so our complexity is equal to $2^{96}$. As we need to store all chains, the precalculation phase has also a cost of $2^{64}$ in memory. However, once the first phase is over, the attacker can perform the attack in only $2^{32}$ operations as she has to calculate only one chain. So, the total cost of the attack is $2^{96}$. The proposed attack satisfies $D T=2^{128}$ as $D=2^{32}$ and $T=2^{96}$. This attack does not improve the complexity of PRINCE given in [7] and [15. However, in our case, $T$ is not the on-line time complexity as it corresponds to the precalculation phase of the attack. Thus, in our attack, we have $D T_{\text {on }}=2^{64}$.

\section{Conclusion}

In this paper, we have presented new tradeoffs for public-key and symmetric-key cryptosystems in the multi-user setting. We have introduced some algorithmic tools for collision-based attacks using the distinguished point technique. The first tool allows to look for the discrete logarithm of $L$ users in parallel using only a $\widetilde{O}(\sqrt{L})$ penalty using random graph process behaviour. The second tool allows to achieve key-recovery of Even-Mansour and related ciphers and is a novel lambda technique to find collisions when two different functions are involved. For the Even-Mansour cipher, we show new tradeoffs that partially solve an open problem due to Dunkelman et al. and we propose an analysis in the multi-user setting. Finally, for the PRINCE cipher, we show generic attacks that improve the best published results in the sense that our time complexity corresponds to a precomputation phase and not to an on-line phase. This last result could also be adapted to similar ciphers such as DESX and would also improve on the best previous attack. 


\section{References}

1. Bernstein, D.J., Lange, T.: Computing Small Discrete Logarithms Faster. In: Galbraith, S., Nandi, M. (eds.) INDOCRYPT 2012. LNCS, vol. 7668, pp. 317338. Springer, Heidelberg (2012)

2. Bernstein, D.J., Lange, T.: Non-uniform Cracks in the Concrete: The Power of Free Precomputation. In: Sako, K., Sarkar, P. (eds.) ASIACRYPT 2013, Part II. LNCS, vol. 8270, pp. 321-340. Springer, Heidelberg (2013)

3. Biham, E.: How to decrypt or even substitute DES-encrypted messages in $2^{28}$ steps. Inf. Process. Lett. 84(3), 117-124 (2002)

4. Biryukov, A., Mukhopadhyay, S., Sarkar, P.: Improved Time-Memory Trade-Offs with Multiple Data. In: Preneel, B., Tavares, S. (eds.) SAC 2005. LNCS, vol. 3897, pp. 110-127. Springer, Heidelberg (2006),

http://dx.doi.org/10.1007/11693383_8

5. Biryukov, A., Shamir, A.: Cryptanalytic Time/Memory/Data Tradeoffs for Stream Ciphers. In: Okamoto, T. (ed.) ASIACRYPT 2000. LNCS, vol. 1976, pp. 1-13. Springer, Heidelberg (2000)

6. Bollobás, B.: Random Graphs, 2nd edn. Cambridge studies in advanced mathematics (2001)

7. Borghoff, J., Canteaut, A., Güneysu, T., Kavun, E.B., Knezevic, M., Knudsen, L.R., Leander, G., Nikov, V., Paar, C., Rechberger, C., Rombouts, P., Thomsen, S.S., Yalçın, T.: PRINCE - A Low-Latency Block Cipher for Pervasive Computing Applications. In: Wang, X., Sako, K. (eds.) ASIACRYPT 2012. LNCS, vol. 7658, pp. 208-225. Springer, Heidelberg (2012)

8. Canteaut, A., Fuhr, T., Gilbert, H., Naya-Plasencia, M., Reinhard, J.-R.: Multiple Differential Cryptanalysis of Round-Reduced PRINCE (Full version). IACR Cryptology ePrint Archive, 2014:89 (2014)

9. Canteaut, A., Naya-Plasencia, M., Vayssière, B.: Sieve-in-the-middle: Improved MITM attacks. In: Canetti, R., Garay, J.A. (eds.) CRYPTO 2013, Part I. LNCS, vol. 8042, pp. 222-240. Springer, Heidelberg (2013)

10. Chatterjee, S., Menezes, A., Sarkar, P.: Another Look at Tightness. In: Miri, A., Vaudenay, S. (eds.) SAC 2011. LNCS, vol. 7118, pp. 293-319. Springer, Heidelberg (2012)

11. Costello, K.P., Vu, V.H.: The rank of random graphs. Random Structures \& Algorithms 33(3), 269-285 (2008)

12. Daemen, J.: Limitations of the Even-Mansour Construction. In: Matsumoto, T., Imai, H., Rivest, R.L. (eds.) ASIACRYPT 1991. LNCS, vol. 739, pp. 495-498. Springer, Heidelberg (1993)

13. Dunkelman, O., Keller, N., Shamir, A.: Minimalism in Cryptography: The EvenMansour Scheme Revisited. In: Pointcheval, D., Johansson, T. (eds.) EUROCRYPT 2012. LNCS, vol. 7237, pp. 336-354. Springer, Heidelberg (2012)

14. Even, S., Mansour, Y.: A construction of a cipher from a single pseudorandom permutation. In: Imai, H., Rivest, R., Matsumoto, T. (eds.) ASIACRYPT 1991. LNCS, vol. 739, pp. 210-224. Springer, Heidelberg (1993)

15. Jean, J., Nikolic, I., Peyrin, T., Wang, L.: S. Wu Security Analysis of PRINCE. In: Fast Software Encryption - 20th International Workshop, FSE 2013, Singapore, March 11-13, 2013. Revised Selected Papers, pp. 92-111 (2013)

16. Kilian, J., Rogaway, P.: How to Protect DES Against Exhaustive Key Search (an Analysis of DESX). J. Cryptology 14(1), 17-35 (2001) 
17. Kuhn, F., Struik, R.: Random walks revisited: Extensions of pollard's rho algorithm for computing multiple discrete logarithms. In: Vaudenay, S., Youssef, A.M. (eds.) SAC 2001. LNCS, vol. 2259, pp. 212-229. Springer, Heidelberg (2001)

18. Lampe, R., Patarin, J., Seurin, Y.: An Asymptotically Tight Security Analysis of the Iterated Even-Mansour Cipher. In: Wang, X., Sako, K. (eds.) ASIACRYPT 2012. LNCS, vol. 7658, pp. 278-295. Springer, Heidelberg (2012)

19. Lee, H.T., Cheon, J.H., Hong, J.: Accelerating ID-based Encryption based on Trapdoor DL using Pre-computation. Cryptology ePrint Archive, Report 2011/187 (2011), http://eprint.iacr.org/

20. Menezes, A.: Another Look at Provable Security. In: Pointcheval, D., Johansson, T. (eds.) EUROCRYPT 2012. LNCS, vol. 7237, pp. 8-8. Springer, Heidelberg (2012)

21. Pollard, J.M.: Kangaroos, Monopoly and Discrete Logarithms. J. Cryptology 13(4), 437-447 (2000)

22. Quisquater, J.-J., Delescaille, J.-P.: How Easy Is Collision Search. New Results and Applications to DES. In: Brassard, G. (ed.) CRYPTO 1989. LNCS, vol. 435, pp. 408-413. Springer, Heidelberg (1990)

23. Soleimany, H., Blondeau, C., Yu, X., Wu, W., Nyberg, K., Zhang, H., Zhang, L., Wang, Y.: Reflection Cryptanalysis of PRINCE-Like Ciphers. In: Fast Software Encryption - 20th International Workshop, FSE 2013, Singapore, March 11-13, 2013. Revised Selected Papers, pp. 71-91 (2013)

24. van Oorschot, P.C., Wiener, M.J.: Parallel Collision Search with Cryptanalytic Applications. J. Cryptology 12(1), 1-28 (1999) 